The commonest tumour in the Kampala Cancer Registry is a lymphosarcoma of young children, first brought into clinical prominence in 1958 by D. Burkitt, ${ }^{3}$ and now called the African lymphoma or the Burkitt tumour. ${ }^{4}$ Yet the usual lymphatic tumours and leukaemias of European adults are not especially common in Uganda. The Burkitt tumour is found almost entirely in children aged 2 to 14 years. It usually presents as a swelling of the jaw, often affecting both mandible and maxilla. The teeth are loosened, and as the orbit is invaded there is progressive exophthalmos, which culminates in the loss of the eye. The first indication may be an abdominal mass, sometimes ovarian or else retroperitoneal, probably due to involvement of the para-aortic lymph nodes. Spread to the vertebral column leads to paraplegia. Occasionally the kidneys, long bones, thyroid, testes, and salivary glands are massively infiltrated. But lesions of the liver, spleen, bone-marrow, and peripheral lymph nodes are much less conspicuous, and the blood picture is not characteristic of leukaemia. Death usually occurs within four to six months, but dramatic improvement may follow methotrexate therapy, and remissions lasting two years have occurred. ${ }^{6}$

Histologically the tumour consists of sheets of small round cells resembling lymphocytes, interspersed among which there are large, pale histiocytes. Tissue culture-studies of the tumour cells suggest that they are lymphoblastic in origin. ${ }^{78}$

This tumour accounts for half the malignant disease of children in Uganda. It was described in the oldest records of Mengo Hospital, though much more about its pathology has been discovered in the last few years. Furthermore, it is not restricted to Uganda, for it is well recognized in most parts of tropical Africa. ${ }^{9}$ So far as is known it appears to be almost entirely confined to Africa, though some cases have been reported in New Guinea. ${ }^{10}$

From the figures of a cancer registry established three years ago G. M. Edington and Catherine $M$. U. Maclean ${ }^{11}$ described in this journal last week the incidence of the Burkitt tumour in Ibadan, Western Nigeria. It accounted for $70 \%$ of all neoplasms in children, and apart from cervical cancer was the commonest malignant disease in Ibadan. The lymphoma is not confined to Negroes, for it has been found in Asian and Eurcpean children also. In a clinicopathological conference reported in the B.M.F. last week $^{12}$ a typical Burkitt tumour with widespread lesions was described in a Lebanese child living in Sierra Leone. The geographical distribution of the disease suggests an arthropod-borne viral aetiology, ${ }^{13}$ but no specific infective agent has yet been isolated.

It is remarkable how well the Mengo records correlate with those of the Kampala Cancer Registry. In his painstaking work Albert Cook not only served the African people, but also laid a corner-stone of the edifice of geographical pathology.

\footnotetext{
1 Davies, J. N. P., Elmes, S., Hutt, M. S. R., Mtimavalye, L. A. R., Owor, R., and Shaper, L., Brit. med. F., 1964, 1, 259.

2 Dodge, O. G., and Linsell, C. A., Cancer, 1963, 16, 1255.

3 Burkitt, D., Brit. F. Surg., 1958, 46, 218.

- Brit. med. f., 1962, 1, 855.

5 Ibid., 1963, 1, 1042.

6 Oettgen, H. F., Burkitt, D., and Burchenal, J. H., Cancer, 1963, 16, 616.

7 Pulvertaft, R. J. V., Lancet, 1964, 1, 238.

- Epstein, M. A., and Barr, Y. M., ibid., 1964, 1, 252.

- Burkitt, D., Brit. med. F., 1962, 2, 1019.

10 O'Conor, G. T., Cancer Res., 1963, 23, 1514. Edington, G. M., and Maclean, C. M. U., Brit. med. F., 1964, 1,

12 Ibid., 1964, 1, 296.

13 Burkitt, D., Ann. roy. Coll. Surg. Engl., 1962, 30, 211.
}

\section{Active Transport}

When carbohydrates are digested they are broken down to monosaccharides. Glycogen, starch, and maltose become glucose, sucrose becomes glucose and fructose, and lactose becomes glucose and galactose. Glucose, galactose, and fructose as well as mannose and pentose are all completely absorbed while the food passes along the small intestines. If their transport across the intestinal mucosa was mere diffusion all should be absorbed at an equal rate. In fact the rate of absorption is different for the various monosaccharides. Their absorption from the peritoneal cavity is by diffusion, but that from the intestines is the same only for pentose. Mannose is absorbed only slightly better by the intestinal mucosa, but fructose is absorbed four times and glucose ten times faster. Galactose is absorbed somewhat faster than glucose. From this we must conclude that the transport of these sugars across the intestinal wall is not governed entirely by physical diffusion as it is from the peritoneum but that a chemical process is at work-“" active transport."

The metabolic processes which provide the energy for this speeding up of absorption must at least in part be the same for the various monosaccharides, for there is evidence of competition between them. For example, the rate of absorption of galactose falls when glucose is administered simultaneously. Phloridzin, which inhibits phosphorylation, inhibits absorption of glucose and galactose. However, phosphorylation by hexokinase cannot be the only mechanism at work, since desoxyglucose is not phosphorylated but it is actively transported, and mannose which is phosphorylated by hexokinase is only little better absorbed by the intestines than by the peritoneum. Potassium has a striking effect on the absorption of glucose from the intestines, and so has thyroxine. Other important catalysts in this context are some of the B vitamins-pantothenic acid, thiamine, and pyridoxine. The diminished absorption of glucose in Addison's disease is now thought not to be related to a specific function of cortical hormones but to be secondary to the low concentration of sodium in plasma and intercellular fluid.

Active transport is responsible for the reabsorption of glucose in the renal tubules. The glomerular filtrate contains glucose at the same concentration as the plasma, yet the urine is normally virtually free of glucose. The function of the glomeruli is to produce a protein-free filtrate of the plasma. The function of the tubular epithelium is to reabsorb more than $99 \%$ of $170-180$ litres of water filtering through the glomeruli per day, and almost all the 170-180 g. of glucose, so that the 24-hour specimen of urine amounts to only $1,500 \mathrm{ml}$. and contains less than $0.5 \mathrm{~g}$. of glucose. In the kidneys, as in the intestines, absorption of glucose is inhibited by phloridzin.

1 Lambert, P. P., in Ciba Foundation Symposium on the Kidney, ed. A. A. G. Lewis and G. E. W. Wolstenholme, 1954. London.

2 Reubi, F. C., ibid.

3 Harris, H., Human Biochemical Genetics, 1959. London.

Penrose, L. S., and Thomas, D. H. H., Ann. hum. Genet., 1959, 23, 442 .

5 Clay, R. D., Darmady, E. M., and Hawkins, M., F. Path. Bact., 1953,

- Stein, W. H., Bearn, A. G., and Moore, S., f. clin. Invest., 1954, 33, 410 .

Dent, C. E., Biochem. F., 1947, 41, 240, and Schweiz. med. Wschr., $1950,80,752$.

Walshe, J. M., Quart. F. Med., 1953, 22, 483.

9 Brit. med. F., 1963, 2, 1148.

Collis, J. E., Levi, A. J., and Milne, M. D., ibid., 1963, 1, 590.

1 See ibid., 1963, 2, 1485.

12 Srikantia, S. G., Venkatachalam, P. S., and Reddy, V., ibid., 1964, 1,

${ }_{13}$ Crowhall, J. C., Scowen, E. F., and Watts, R. W. E., ibid., 1963, 1, 588. 
Abnormal amounts of glucose in the urine can be due to two principal causes. When the level of blood sugar is abnormally high a raised amount of glucose enters the tubules and the mechanism of active transport is overtaxed. This happens in diabetes mellitus or when glucose is too quickly absorbed as in hyperthyroidism, after gastrectomy, or when adrenaline causes an unduly rapid conversion of glycogen in the liver to glucose. Alternatively the blood-sugar level and the glucose concentration in the glomerular filtrate may be normal but the tubular reabsorption is lower than normal. This second type of glycosuria is collectively named renal glycosuria. It may occur in some diseases of the kidney, and in other persons it may arise from an error of metabolism in the tubular epithelium, often genetically determined. This functional defect can be due to a general reduction in the reabsorptive capacity of all the nephrons, or individual nephrons may vary widely in efficiency. ${ }^{1-4}$ In the first type the mean renal threshold is below the normal level of $250 \mathrm{mg}$. glucose per $100 \mathrm{ml}$. plasma. In the second type the mean threshold may be normal but the minimal threshold is lowered, so that the level at which some of the nephrons are unable to reabsorb glucose efficiently lies below the minimal threshold of normal persons, which is $170 \mathrm{mg}$. per $100 \mathrm{ml}$.

The type of renal glycosuria in which the mean threshold is lower than normal and reabsorptive capacity is diminished throughout all the nephrons could arise because smaller amounts of the metabolites responsible for active transport are produced, or because one of the enzymes utilizing these metabolites is deficient. The defect is often specific for glucose, and other metabolites are not excreted abnormally. Hence it is unlikely that an anatomical abnormality of the nephrons can be the cause. In the type of renal glycosuria with lowered minimal threshold but normal mean threshold the cause may also be that metabolites or enzymes are deficient in some nephrons and not in others, but $\mathrm{H}$. Harris ${ }^{3}$ has drawn attention to the possibility that in some cases many of the nephrons may be abnormal in structure-for instance, in having unduly short tubules.

While renal glycosuria is usually not associated with increased excretion of other metabolites in the urine, some rare conditions are known, usually genetic, in which it is. In the Toni-Fanconi syndrome many amino-acids appear in the urine besides glucose and phosphate. Here microdissection of nephrons has shown ${ }^{5}$ that the proximal tubules are shortened and joined to the glomeruli by a narrow "swan's neck." In Wilson's disease there is a deficiency of caeruloplasmin-the plasma protein that carries copper. Some of the excess inorganic copper of the plasma is deposited in the tubules and upsets their function. ${ }^{6}$ Glycosuria and a general amino-aciduria are the outcome. In both the Toni-Fanconi syndrome and Wilson's disease the levels of blood sugar and amino-acids in the plasma are normal, and the defect is clearly related to a failure of the renal tubules to reabsorb them.

Most of the studies of active transport have in the past been concerned with glucose and other monosaccharides. Now at page 327 of the B.M.F. this week Professor M. D. Milne surveys in his Bradshaw lecture much of what we know about the active transport of the amino-acids. Satisfactory investigation of the urinary amino-acids dates only from the time when C. E. Dent ${ }^{7}$ began to apply paper chromatography to their determination.

As in glycosuria so in amino-aciduria : two types exist. One is the overflow type, in which amino-acids are at an abnormally high concentration in the plasma. The other is the renal type, in which the levels in the plasma are normal but tubular reabsorption is deficient.

The amino-aciduria of liver failure belongs to the first group and corresponds to the glycosuria of diabetes mellitus. ${ }^{8}$ The liver cannot metabolize amino-acids, and high levels are found in the plasma together with amino-aciduria. Alternatively, the plasma levels of only some amino-acids may be raised. Though these amino-acids are normally reabsorbed in the tubules by active transport, their levels in the plasma rise above that with which the tubules are normally required to cope. This happens in some inborn errors of metabolism, of which the best-known example is phenylketonuria. Phenylalanine cannot be converted to tyrosine and becomes phenylpyruvic acid. Besides phenylpyruvic acid its precursor (phenylalanine) and a breakdown product (phenyllactic acid) are found in the urine. Amino-aciduria may also be due to the release from cells into plasma of amino-acids which are constituents normally confined to the cells. No active transport mechanism is available for these amino-acids, and when they are presented to the tubules they are summarily excreted more or less at the same rate as they filter through the glomeruli. One of these "overflow amino-acidurias" is cystathioninuria, recently reviewed in these columns. ${ }^{9}$

Renal amino-aciduria due to a failure of active transport may originate in generalized tubular damage, as in ToniFanconi's syndrome or in Wilson's disease. Alternatively it may be restricted to certain types of amino-acids. Such a specific tubular defect is the cause of cystinuria, ${ }^{10}$ and perhaps homocystinuria. ${ }^{11}$ Attention has been focused on cystine because this amino-acid has a remarkably low solubility and increased levels in the urine are the cause of nephrolithiasis. But in fact cystinuria is a defect of reabsorption of all dibasic amino-acids, and besides cystine the urine contains lysine, arginine, and ornithine, as well as (from a reaction between cystine and homocysteine) the disulphide of cysteine and homocysteine. In Hartnup's disease, a variant of which was reported in this journal last week, ${ }^{12}$ the defect lies in the active transport of mono-aminomonocarboxylic acids, such as alanine, serine, leucine, phenylalanine, and tryptophan. The amino-aciduria is of no clinical consequence, but the associated failure of active transport across the intestinal mucosa may cause severe deficiency states. Attempts to convert the patient's cystine to the more soluble penicillamine cysteine sulphide ${ }^{13}$ may reduce the clinical severity of this inborn error in the active transport of the dibasic amino-acids, and Professor Milne discusses possibilities of treating Hartnup's disease, the studies of which are hampered by its rarity. As he points out, the diseases of amino-acid transport are of greater interest than their rarity would suggest at first sight as being appropriate, for they increase our understanding of important physiological principles. Moreover in recent years the gain in knowledge has led to more effective therapy.

\section{Struggle for Cleaner Air}

The two most refractory environmental problems which seriously affect health are slums and atmospheric pollution. At the present time the Government is making determined efforts to reduce both these evils. While every country in the world has slums, many much worse than those in Britain, our problem of urban smoke pollution must be second to none. 